\title{
First Keck Interferometer measurements in self-phase referencing mode: spatially resolving circum-stellar line emission of 48 Lib
}

J-U. Pott, J. Woillez, S. Ragland, P. L. Wizinowich, J. A. Eisner, et al.

J-U. Pott, J. Woillez, S. Ragland, P. L. Wizinowich, J. A. Eisner, J. D. Monnier, R. L. Akeson, A. M. Ghez, J. R. Graham, L. A. Hillenbrand, R. Millan-Gabet, E. Appleby, B. Berkey, M. M. Colavita, A. Cooper, C. Felizardo, J. Herstein, M. Hrynevych, D. Medeiros, D. Morrison, T. Panteleeva, B. Smith, K. Summers, K. Tsubota, C. Tyau, E. Wetherell, "First Keck Interferometer measurements in self-phase referencing mode: spatially resolving circum-stellar line emission of 48 Lib," Proc. SPIE 7734 Optical and Infrared Interferometry II, 77340P (21 July 2010); doi:

$10.1117 / 12.856922$

Event: SPIE Astronomical Telescopes + Instrumentation, 2010, San Diego, California, United States 


\title{
First Keck interferometer measurements in self-phase referencing mode: spatially resolving circum-stellar line emission of $48 \mathrm{Lib}$
}

\author{
J.-U. Pott ${ }^{a, b, c}$, J. Woillez ${ }^{a}$, S. Ragland ${ }^{a}$, P. L. Wizinowich ${ }^{a}$, J. A. Eisner ${ }^{d}$, J. D. Monnier ${ }^{e}$, R. L. \\ Akeson $f$, A. M. Ghez ${ }^{c, g}$, J. R. Graham ${ }^{h}$, L. A. Hillenbrand ${ }^{i}$, R. Millan-Gabet ${ }^{f}$, E. Appleby ${ }^{a}$, B. \\ Berkey $^{a}$, M. M. Colavita ${ }^{j}$, A. Cooper ${ }^{a}$, C. Felizardo $f$, J. Herstein $f$, M. Hrynevych ${ }^{a}$, D. Medeiros ${ }^{a}$, D.

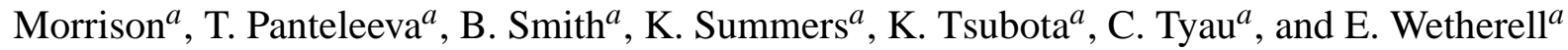 \\ ${ }^{a}$ Max-Planck-Institut für Astronomie, Königstuhl 17, D-69117 Heidelberg, Germany; \\ ${ }^{b}$ W. M. Keck Observatory, California Association for Research in Astronomy, Kamuela, HI 96743, \\ USA; \\ ${ }^{c}$ Div. of Astronomy \& Astrophysics, University of California, Los Angeles, CA 90095-1547, USA; \\ ${ }^{d}$ Steward Observatory, University of Arizona, Tucson, AZ 85721, USA; \\ ${ }^{e}$ Astronomy Department, University of Michigan, Ann Arbor, MI 48109, USA; \\ ${ }^{f}$ NASA Exoplanet Science Institute, Caltech, Pasadena, CA 91125, USA; \\ ${ }^{g}$ Institute of Geophysics and Planetary Physics, University of California, Los Angeles, CA \\ 90095-1565, USA; \\ ${ }^{h}$ Astronomy Department, University of California Berkeley, CA 94720, USA; \\ ${ }^{i}$ California Institute of Technology, Pasadena, CA 91125, USA; \\ ${ }^{j}$ Jet Propulsion Laboratory, California Institute of Technology, Pasadena, CA 91109, USA
}

\begin{abstract}
Recently, the Keck interferometer was upgraded to do self-phase-referencing (SPR) assisted K-band spectroscopy at $R \sim$ 2000. This means, combining a spectral resolution of $150 \mathrm{~km} / \mathrm{s}$ with an angular resolution of 2.7 mas, while maintaining high sensitiviy. This SPR mode operates two fringe trackers in parallel, and explores several infrastructural requirements for off-axis phase-referencing, as currently being implemented as the KI-ASTRA project. The technology of self-phasereferencing opens the way to reach very high spectral resolution in near-infrared interferometry. We present the scientific capabilities of the KI-SPR mode in detail, at the example of observations of the Be-star $48 \mathrm{Lib}$. Several spectral lines of the cirumstellar disk are resolved. We describe the first detection of Pfund-lines in an interferometric spectrum of a Be star, in addition to $B r \gamma$. The differential phase signal can be used to (i) distinguish circum-stellar line emission from the star, (ii) to directly measure line asymmetries tracing an asymetric gas density distribution, (iii) to reach a differential, astrometric precision beyond single-telescope limits sufficient for studying the radial disk structure. Our data support the existence of a radius-dependent disk density perturbation, typically used to explain slow variations of Be-disk hydrogen line profiles.
\end{abstract}

Keywords: stars: emission-line, Be circumstellar matter stars: individual (48 Lib) techniques: interferometric techniques: spectroscopic infrared: stars

\section{INTRODUCTION}

The ASTRA (ASTrometric and phase-Referencing Astronomy) upgrade program aims at extending the sensitivity and spectral resolution of the Keck interferometer (KI) through phase referencing. ${ }^{1-4}$ The data in this article were observed as a science demonstration project of the self-phase-referencing (SPR, on-axis fringe tracking) mode. ${ }^{5,6}$ Due to the stabilized fringes, $>100 \mathrm{x}$ longer integrations can be taken on the second fringe camera. SPR offers a spectral resolution $R(=\lambda / \Delta \lambda)$ of 2000, which is Nyquist sampled at $\mathrm{R}=1000$ (330 pixels across the $\mathrm{K}^{\prime}$-band) at $\mathrm{K} \leq 7$ mag. ${ }^{5}$ The here presented data demonstrate that the differential visibility phase can be retrieved at unprecedented precision ( $3 \mathrm{mrad}$ at $K=5 \mathrm{mag}$, which represents $3 \mu$ as on sky or a centroiding precision of $10^{-3}$ ). Non-linear phase changes in the source spectrum can be measured, and typically indicate translations of the photo-center over the respective spectral channels on the sky. The

Send correspondence to J.-U. Pott - E-mail: jpott@ mpia.de, Telephone: +49-6221-528-0

Optical and Infrared Interferometry II, edited by William C. Danchi, Françoise Delplancke, Jayadev K. Rajagopal, Proc. of SPIE Vol. $7734,77340 P$ - (c) 2010 SPIE · CCC code: 0277-786X/10/\$18 · doi: 10.1117/12.856922 
operational readiness of KI-SPR was successfully demonstrated with the here discussed observations of 48 Lib on the night of April 25, 2008 (UT). In addition, several young stellar objects were observed successfully. ${ }^{7}$ We show at the example of $48 \mathrm{Lib}$ the wealth of spatial and spectral information that can be provided by SPR data.

\section{BE-STAR DISK AS SPR SCIENCE CASE}

Since early on, it is known that a large fraction of Be stars show cyclic variations in the ratio between the violet and red flux of the HI emission lines (V/R). ${ }^{8}$ Early measurements with the GI2T interferometer spatially and timely resolved the $H \alpha$ emission of $\gamma$-Cas, showing spatial variation of the line emission region with time. ${ }^{9}$ Theoretical models predict a prograde one-armed spiral density pattern, slowly precessing in the disk and creating the V/R cycles. ${ }^{10,11}$ The latter authors calculate that the radial extension of such a disk oscillation mode should be confined to a few stellar radii, which can be probed by interferometry. Spectro-interferometric observations of the photocenter shift in hydrogen lines of $\zeta$ Tau appear to directly confirm a one-armed density mode in the disk. ${ }^{12-14}$

Goal of the science demonstration project was to show, that KI-SPR data are suitable to measure directly, and efficiently, even in a single dataset, core parameters like radial extent and radius-dependence of the often discussed slowly rotating density inhomogeneities in the gas around Be stars. We chose $48 \mathrm{Lib}$ as science demonstration target because of its edge-on disk geometry. This ensures strong features in the differential visibility and phase signals across the emission lines.

\section{RESULTS}

Each dataset consists of 155 frames of $0.5 \mathrm{sec}$ integration time. The resulting measurables of the SPR observation (flux, $V^{2}$, and $d \phi$ ) are shown in Fig. 1. Spectroscopic observations at this resolution are a new feature at KI. ${ }^{15}$ To ensure proper wavelength calibration, the slope of the spectrometer wavelength table is measured internally by a Fourier transform based technique. In the standard KI observing setup, this slope is applied automatically to the data provided through the NExScI archive. Furthermore, we used telluric absorption lines in the raw spectra to estimate an offset in the wavelength calibration (typically of order of a few tenths of one spectral pixel).

The quality of the resulting wavelength calibration is shown by overplotting the HI-recombination line centers in Fig. 1 (dotted lines). It is reassuring, that (1) the flux line profiles are centered, that (2) the various Pfund lines show the same line profile, and most importantly, that (3) the $d \phi$ of both $B r \gamma$ and Pfund lines crosses zero close to the transition wavelengths, and with the same sign of the slope. (3) is expected for an inclined Keplerian disk.

\subsection{Data quality}

To show the high differential stability of the data over the used band, linear continua are shown in red. The red line in the $d \phi$ panel marks zero phase, and is not a fit. The resulting pixel-to-pixel errors are $\Delta$ flux $=0.003, \Delta V^{2}=0.007$, and $\Delta d \phi=3 \mathrm{mrad}$. The higher relative precision of the $d \phi$ signal with respect to the precision of the visibility is expected for such spectro-interferometric measurements. ${ }^{16}$

This differential stability at the level of a few $10^{-3}$ is unprecedented for spectrally dispersed OLBI data at the spectral resolution and sensitivity offered by KI-SPR. It is the key to derive the scientific results presented in the next section. The stable $d \phi$ slope was achieved by two steps in the data reduction, which reflect that a non-zero group delay creates a quadratic phase slope over the $K$-band, due to the use of airfilled delay lines. ${ }^{17}$ First, frames with group delays of more than $4.5 \mu \mathrm{m}$ are rejected. The remaining set of frames (> 90\%) was corrected for the linear phase slope of the group delay. In a second step, the quadratic phase slope due to the group delay was fitted, and removed. These pre-processed frames were averaged, and the standard deviation describes the differential precision well. More details on the data reduction and calibration are given in. ${ }^{6}$ The 3 mrad precision level of $d \phi$ is given by the SNR. Fainter sources will have lower precision. Longer integrations will probably improve on the phase precision.

\subsection{Information on the radial disk structure}

Fig. 1 shows that different components have been detected in the spectra of 48 Lib: a clearly resolved continuum emission, and $\mathrm{HI}$ recombination line emission of $\mathrm{Br} \gamma$ and, for the first time, various Pfund lines. Our quantitative results are summarized in Table 1. $\theta_{\text {cont }}^{\mathrm{FWH}}$ and $\theta_{\text {cont,disk }}^{\mathrm{FWHM}}$ give the Gaussian full-width-half-maximum, fitted to the continuum visibilites without, and with taking into account the photospheric contribution, respectively. $F_{\text {line }}$ and $F_{\text {line }}^{\text {corr }}$ denote the total and correlated line fluxes. The violet $(\mathrm{V})$ and red $(\mathrm{R})$ velocity bins are given as well as the respective $\mathrm{V} / \mathrm{R}$ values. $\theta_{\text {line }}^{\mathrm{FWHM}}$ and $P C$ 


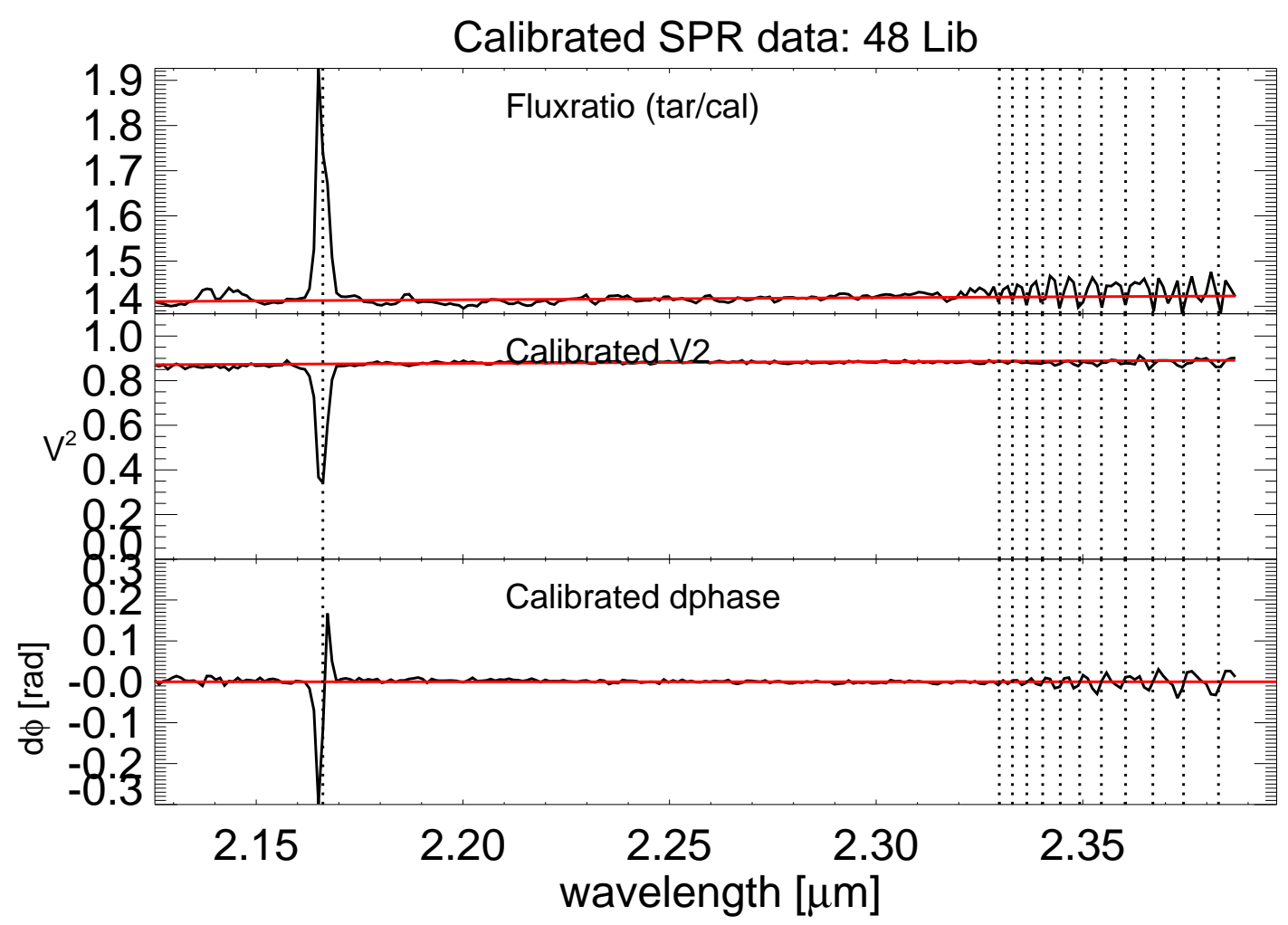

Figure 1. Top: Mean calibrated flux ratio between the $48 \mathrm{Lib}$ and the continuum divided calibrator. The red solid line marks a linear continuum fit. The different line profiles of $B r \gamma$ and $P f$-emission lines are clearly visible Center: Mean calibrated $V^{2}$ of 48 Lib showing that both the NIR continuum and the recombination line emission are spatially resolved by the interferometer. A linear continuum (red line) was fitted to the line free regions. Bottom: The calibrated differential phase data. The red line marks zero phase. All plots show the rest wavelength of the target. The vertical dotted lines indicate the rest wavelength of the recombination lines. It is apparent that all lines show the same slope at the line center, as expected for disk emission. 
Table 1: Measured and derived properties of the circumstellar shell of 48 Lib. See Sect. 3.2

\begin{tabular}{|c|c|c|c|}
\hline$K_{\text {cont }}^{\prime}$ (Gauss-disk only): & $\theta_{\text {cont }}^{\mathrm{FWHM}}$ & $0.94 \pm 0.03$ mas & \\
\hline$K_{\text {cont }}^{\prime}($ Gauss-disk + star $):$ & $\theta_{\text {cont,disk }}^{\mathrm{FWHM}}$ & $1.65 \pm 0.05 \mathrm{mas}$ & \\
\hline$B r \gamma$ & $V_{\left(-250,-100 \mathrm{~km} \mathrm{~s}^{-1}\right)}$ & $R_{\left(100,250 \mathrm{~km} \mathrm{~s}^{-1}\right)}$ & $V / R$ \\
\hline$F_{\text {line }}($ norm. $)$ & $0.29 \pm 0.01$ & $0.16 \pm 0.01$ & $1.80 \pm 0.2$ \\
\hline$F_{\text {line }}^{\text {corr }}($ norm. $)$ & $0.23 \pm 0.01$ & $0.14 \pm 0.01$ & $1.66 \pm 0.2$ \\
\hline$\theta_{\text {line }}^{\mathrm{FWHM}}$ (mas) & $1.7 \pm 0.2$ & $1.4 \pm 0.2$ & $1.2 \pm 0.2$ \\
\hline$P C_{\text {line }}^{\text {shift }}$ (mas) & $2.2 \pm 0.2$ & $1.9 \pm 0.2$ & $1.2 \pm 0.2$ \\
\hline$P f_{\text {avg }}(5: 24 . .28)$ & $V_{\left(-325,-175 \mathrm{~km} \mathrm{~s}^{-1}\right)}$ & $R_{\left(175,325 \mathrm{~km} \mathrm{~s}^{-1}\right)}$ & $V / R$ \\
\hline$F_{\text {line }}($ norm. $)$ & $0.029 \pm 0.01$ & $0.028 \pm 0.01$ & $1.0 \pm 0.1$ \\
\hline$F_{\text {line }}^{\text {corr }}($ norm.) & $0.024 \pm 0.01$ & $0.024 \pm 0.01$ & $1.0 \pm 0.1$ \\
\hline$\theta_{\text {line }}^{\mathrm{FWHM}}(\mathrm{mas})$ & $1.9 \pm 0.3$ & $1.5 \pm 0.2$ & $1.2 \pm 0.3$ \\
\hline$P C_{\text {line }}^{\text {shift }}($ mas $)$ & $0.9 \pm 0.3$ & $0.9 \pm 0.3$ & $1.1 \pm 0.3$ \\
\hline
\end{tabular}

shift are the Gaussian FWHM fit and intrinsic photocenter shift of the line in the indicated velocity bin. All uncertainties
line given in the table are derived from the standard deviations of the mean total flux and correlated flux spectra shown in the figures. These uncertainties reflect the differential, relative precision of the values.

To improve on the signal to noise of the Pfund lines, we use a flux-weighted average of the five strongest Pfund lines (24..28) with a signal-to-noise ratio of 5-8. It appears that $d \phi$ derived photocenter offsets of the Pfund-lines are significantly smaller than the $B r \gamma$ offsets, while the velocities are higher. In the Keplerian disk picture, this hints to the fact that the majority of the Pfund emission originates from significantly smaller centro-stellar radii than the $B r \gamma$ emission. This finding confirms the idea of optically thick hydrogen emission in Be-star disks. ${ }^{18}$ Comparing the respective Einstein absorption coefficients, we find $B_{5,24}<B_{4,7}<B_{2,3}$, and can expect that the Pfund emission is emitted from inside of $B r \gamma$ as seen in the data. With the same reasoning, $B r \gamma$ should be emitted at smaller stellocentric radii than $H_{\alpha}$, which was indirectly confirmed by the data of ${ }^{19}$ (similarly for $\zeta$ Tau $^{13}$ ). Also the smaller $H_{\alpha}$ peak velocities ${ }^{19}$ are consistent with larger stellocentric radii compared to $\mathrm{Br} \gamma$ and Pfund lines.

In addition to this radial information, the overall line profiles of the detected $B r \gamma$ and Pfund emission are different. The blue-shifted $B r \gamma$ emission is significantly stronger than the red part, while the average Pfund profile is symmetric. The $H \alpha$ spectra of $48 \mathrm{Lib}$, provided by the BeSS database * also report a V/R $>1$ at the time of our observations. Such a radius dependent line emission asymmetry (from $H \alpha$ to Pfund) reminds of the often observed phase shifts of the slow, but periodic V/R variations of different hydrogen lines of Be-stars. ${ }^{13,19}$ Although our single dataset cannot reveal a proper spiral density wave in the disk as predicted by theoretical models, ${ }^{10,11}$ the linear constraints from the KI data are consistent with those models, and rule out scenarios of dominating hydrogen emission signifcantly outside of $18(\mathrm{Br} \gamma)$ and 8 (Pfund) stellar radii. The continuum emission by bound-free and free-free thermal emission in the disk is clearly resolved. The data suggest similar centro-stellar radii than for the Pfund emission lines.

This discussion demonstrates how the combination of total, and correlated flux and $d \phi$ measurements at the differential precision of KI-SPR give strong linear constraints on the disk structure, in particular if several lines can be resolved. ${ }^{13}$

${ }^{*}$ http://basebe.obspm.fr/basebe/ 


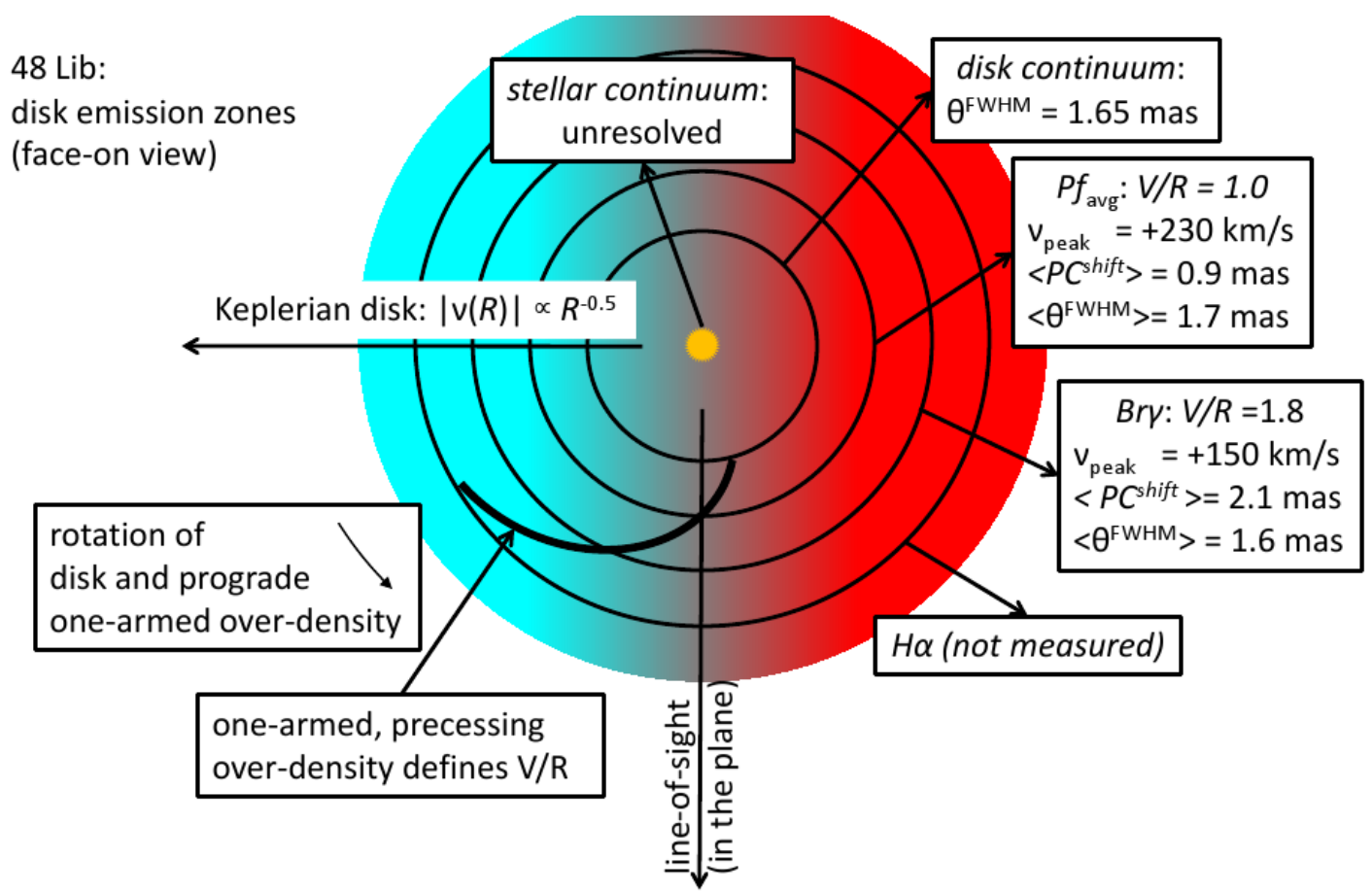

Figure 2. Visualization of the measured disk properties (not to scale). The shown one-armed over-density pattern would explain the measured $V / R$ and correlated flux profiles of $B r \gamma$ and $P f_{\text {avg }}$, as predicted by, ${ }^{11}$ but the here shown pattern is not based on a model calculation. The exact shape of the pattern is not constrained by a single KI-SPR dataset. The relative location of the optical $H \alpha$ line is added for completeness, matching previous, single telescope velocity and $V / R$ measurements. ${ }^{19}$

discuss how such constraints can be modelled in detail for the disk of a Be star. Without applying such a physical model, we simply depict in Fig. 2 that a one-armed overdensity could explain all measured disk parameters.

\section{OUTLOOK}

Repeated KI-SPR observations with the used setup of $48 \mathrm{Lib}$, or similar Be stars, would show if the measured line properties would change with time according to a precessing density perturbation, as the cyclic $\mathrm{V} / \mathrm{R}$ variations suggest. In principle, using different dispersive elements, the self-phase-referencing technique would allow to achieve even higher spectral resolution.

We demonstrated that spectro-interferometric astrometry in the $K^{\prime}$-band can produce extremly accurate results on the 5-10 $\mu$ as level. The detection of the Pfund lines shows that even weak line emission can produce a significant signal in the differential phase. Covering large parts of the $K^{\prime}$-band allows for the simulatenous detection of several emission lines in one spectrum with KI-SPR. The is ideal for differential studies of circum-stellar disks, emission line binaries, and other objects resolved by the $85 \mathrm{~m}$ baseline of the KI. Extragalactic targets, like the kinematics of the broad-line region of nearby AGN, could be directly resolved with such spectrally resolved interferometric observations, if the current limiting magnitude of KI-SPR could be pushed to the $K \sim 9-10 \mathrm{mag}$ level.

\section{ACKNOWLEDGMENTS}

The realization of the KI-ASTRA upgrade is supported by the NSF MRI grant, AST-0619965. The data presented herein were obtained at the W.M. Keck Observatory, which is operated as a scientific partnership among the California Institute of Technology, the University of California and the National Aeronautics and Space Administration. The Observatory was made possible by the generous financial support of the W.M. Keck Foundation. The authors wish to recognize and acknowledge the very significant cultural role and reverence that the summit of Mauna Kea has always had within the 
indigenous Hawaiian community. We are most fortunate to have the opportunity to conduct observations from this mountain. The Keck Interferometer is funded by the National Aeronautics and Space Administration as part of its Exoplanet Exploration program. This research has made use of the SIMBAD database, operated at CDS, Strasbourg, France. This research has made use of NASAs Astrophysics Data System Bibliographic Services. This work has made use of the BeSS database, operated at GEPI, Observatoire de Meudon, France.

\section{REFERENCES}

[1] Wizinowich, P., a. G. J. and Woillez, J. e. a., “NSF-MRI Award Abstract \#0619965,” (2007).

[2] Pott, J.-U. and Woillez, J. e. a., "Astrometry with the Keck-Interferometer: the ASTRA project and its science," in [Proceedings of the summerschool Astrometry and Imaging with the Very Large Telescope Interferometer, June, 2008, Keszthely, Hungary, in press ], arXiv:0811.2264 (2008).

[3] Woillez, J. M. e. a., "ASTRA: ASTrometry and phase-Referencing Astronomy on the Keck interferometer," Presented at this SPIE conference 7734-37 (2010).

[4] Eisner, J. A. e. a., "Science with the Keck Interferometer ASTRA Program," Presented at this SPIE conference 7734-36 (2010).

[5] Woillez, J. M. e. a., "Self-phase-referencing for high spectral resolution with the Keck interferometer - Phase I of the ASTRA project," PASP (to be submitted) (2010).

[6] Pott, J.-U. e. a., "Probing local density inhomogeneities in a circum-Be-star disk using the new spectro-astrometry at the Keck interferometer," ApJ (submitted) (2010).

[7] Eisner, J. A. e. a., "Spatially and Spectrally Resolved Hydrogen Gas within 0.1 AU of T Tauri and Herbig Ae/Be Stars," ApJ (submitted) (2010).

[8] Curtiss, R. H., "Studies of spectrum variables of Class Be (abstract)," Popular Astronomy 33, 537-+ (1925).

[9] Mourard, D., Bosc, I., Labeyrie, A., Koechlin, L., and Saha, S., "The rotating envelope of the hot star Gamma Cassiopeiae resolved by optical interferometry," Nature 342, 520-522 (Nov. 1989).

[10] Okazaki, A. T., "Long-term V/R variations of Be stars due to global one-armed oscillations of equatorial disks," PASJ 43, 75-94 (Feb. 1991).

[11] Papaloizou, J. C., Savonije, G. J., and Henrichs, H. F., “On the long-term periodicities in Be stars,” AEA 265, L45L48 (Nov. 1992).

[12] Vakili, F., Mourard, D., Stee, P., Bonneau, D., Berio, P., Chesneau, O., Thureau, N., Morand, F., Labeyrie, A., and Tallon-Bosc, I., "Evidence for one-armed oscillations in the equatorial disk of zeta Tauri from GI2T spectrally resolved interferometry," AEA 335, 261-265 (July 1998).

[13] Carciofi, A. C., Okazaki, A. T., Le Bouquin, J., Štefl, S., Rivinius, T., Baade, D., Bjorkman, J. E., and Hummel, C. A., "Cyclic variability of the circumstellar disk of the Be star $\zeta$ Tauri. II. Testing the 2D global disk oscillation model," AEA 504, 915-927 (Sept. 2009).

[14] Štefl, S., Rivinius, T., Carciofi, A. C., Le Bouquin, J., Baade, D., Bjorkman, K. S., Hesselbach, E., Hummel, C. A., Okazaki, A. T., Pollmann, E., Rantakyrö, F., and Wisniewski, J. P., "Cyclic variability of the circumstellar disk of the Be star $\zeta$ Tauri. I. Long-term monitoring observations," AEA 504, 929-944 (Sept. 2009).

[15] Ragland, S. e. a., "Recent progress at the Keck Interferometer," Presented at this SPIE conference 7734-1 (2010).

[16] Millour, F., Vannier, M., Petrov, R. G., Lopez, B., and Rantakyrö, F., "Extrasolar Planets with AMBER/VLTI, What can we expect from current performances?," in [IAU Colloq. 200: Direct Imaging of Exoplanets: Science $\mathcal{E}$ Techniques ], C. Aime \& F. Vakili, ed., 291-296 (2006).

[17] Colavita, M. M., Wallace, J. K., Hines, B. E., Gursel, Y., Malbet, F., Palmer, D. L., Pan, X. P., Shao, M., Yu, J. W., Boden, A. F., Dumont, P. J., Gubler, J., Koresko, C. D., Kulkarni, S. R., Lane, B. F., Mobley, D. W., and van Belle, G. T., "The Palomar Testbed Interferometer," ApJ 510, 505-521 (Jan. 1999).

[18] Lenorzer, A., de Koter, A., and Waters, L. B. F. M., "Hydrogen infrared recombination lines as a diagnostic tool for the geometry of the circumstellar material of hot stars," AEA 386, L5-L8 (May 2002).

[19] Wisniewski, J. P., Kowalski, A. F., Bjorkman, K. S., Bjorkman, J. E., and Carciofi, A. C., "Toward Mapping the Detailed Density Structure of Classical Be Circumstellar Disks,” ApJ 656, L21-L24 (Feb. 2007). 